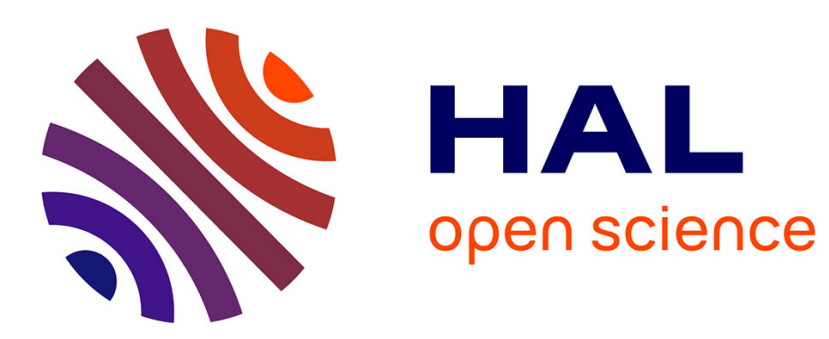

\title{
Une écologie symbolique totonaque. Le municipe de Huehuetla (Puebla, Mexique)
}

Nicolas Ellison

\section{To cite this version:}

Nicolas Ellison. Une écologie symbolique totonaque. Le municipe de Huehuetla (Puebla, Mexique). Journal de la Société des américanistes, 2004, 90 (2), https://jsa.revues.org/1332. 10.4000/jsa.1332 . hal-01285835

\section{HAL Id: hal-01285835 \\ https://hal.science/hal-01285835}

Submitted on 15 Mar 2016

HAL is a multi-disciplinary open access archive for the deposit and dissemination of scientific research documents, whether they are published or not. The documents may come from teaching and research institutions in France or abroad, or from public or private research centers.
L'archive ouverte pluridisciplinaire HAL, est destinée au dépôt et à la diffusion de documents scientifiques de niveau recherche, publiés ou non, émanant des établissements d'enseignement et de recherche français ou étrangers, des laboratoires publics ou privés. 


\title{
Journal de la société des américanistes
}

2004, 90-2

Articles

\section{Une écologie symbolique totonaque. Le municipe de Huehuetla (Puebla, Mexique)}

NiCOLAS ELLISON

p. $35-62$

\author{
Abstracts \\ Français English Español \\ Une écologie symbolique totonaque. Le municipe de Huehuetla (Puebla, Mexique). Cet article montre comment, \\ dans le contexte de changements écologiques et de tensions interethniques régionales, les représentations cosmologiques - et \\ notamment le symbolisme sylvestre - ont pris une nouvelle signification à partir des contrastes d'usage de l'environnement entre \\ Totonaques et Métis. Les Totonaques s’identifient par leur relation privilégiée aux espaces boisés ou agroforestiers en mobilisant \\ le système classificatoire chaud/froid. Ce principe dualiste est, comme on le sait, fondamental dans le domaine des pratiques \\ thérapeutiques et culinaires, mais son application à la répartition symbolique des espaces est encore peu étudiée dans l'aire \\ mésoaméricaine.
}


A symbolic ecology of the Totonacs. The municipio of Huehuetla (Puebla, Mexico). This paper shows how, in the present context of ecological change and regional interethnic tensions, cosmological representations and especially forest symbolism find a new meaning in the contrast between Totonac found Mestizo use of the environment. Totonac found their identity on their special relation with wooded or agro-forestry areas, using a hot/cold classificatory system. Scholars have often focused on this dualistic principle in therapeutic and culinary practices, but its application at the level of symbolic classification of ecological spaces has rarely been studied in the Mesoamerican area.

Una ecología simbólica totonaca. El municipio de Huehuetla (Puebla, México). Este artículo muestra cómo, en el contexto de cambios ecológicos y de tensiones interétnicas regionales, las representaciones cosmológicas y especialmente el simbolismo de la selva, toman una nueva significación a partir de los contrastes en los usos de los recursos naturales entre totonacas y mestizos. Los totonacas se identifican por su relación privilegiada con los espacios silvestres o agroforestales, movilizando para ello el sistema clasificatorio de la polaridad frío/caliente. Este principio dualista es, como cada uno sabe, fundamental en lo referente a las prácticas medicinales y culinarias, pero su aplicación a la repartición simbólica de los espacios ha sido poco estudiada para el área mesoamericana.

\section{Index terms}

Keywords : caféiculture, forêt, polarité chaud/froid, représentations écologiques Keywords : coffee-orchards, ecological representations, forest, hot/cold polarity

Palabras claves : cafeicultura, religión, monte, polaridad frío/caliente, representaciones ecológicas

Geographical index : Huehuetla, Mésoamérique, Puebla, Mexique, Totonaques

Subject index : Ethnologie

\section{Editor's notes}

Manuscrit reçu en octobre 2003, accepté pour publication en septembre 2004

\section{Full text}

« Les Métis n'aiment pas les arbres », « là, où il y a de grands arbres, on voit la joie de la terre »... ces phrases prononcées par mes hôtes totonaques mettent en avant le rapport privilégié qui existe entre eux et les espaces boisés dans un contexte écologique où les caféières et les pâturages ont remplacé la forêt. Recueillis au cours de l'enquête de terrain qui était axée sur les pratiques agricoles, ces propos invitent à s'interroger, de manière plus systématique, sur le rôle du symbolisme sylvestre et son réinvestissement dans la construction de l'appartenance locale chez les Indiens totonaques de Huehuetla, dans le sud de la Sierra de Puebla ${ }^{1}$.

2 Dans un texte programmatique, Appadurai (1995, p. 205) propose d'analyser les usages agro-écologiques comme des " techniques for the spatial production of locality ». Conformément à cette approche, nous tenterons, dans cet article, de montrer à partir des contrastes entre l'utilisation des ressources naturelles par les Totonaques et celle des Métis comment les représentations cosmologiques liées à l'environnement, et notamment le symbolisme sylvestre, prennent une nouvelle signification dans un contexte de changements écologiques et de tensions interethniques régionales.

3 Les communautés totonaques et nahua de la Sierra de Puebla sont aujourd'hui pleinement insérées dans 
l'économie nationale et internationale, notamment grâce à la production et la commercialisation du café2 ${ }^{2}$ Elles développent des liens d'interdépendance complexes avec les centres urbains, surtout en raison des migrations pendulaires des jeunes vers les grandes villes du centre du Mexique (Puebla, Mexico). Les activités agricoles, et notamment celles liées aux cycles du maïs, n'en occupent pas moins une place prépondérante dans la vie sociale.

$4 \quad$ Sur le plan de la politique régionale, le pouvoir exercé par la minorité métisse sur les chefs-lieux des municipes de la région a été remis en question, au cours des deux dernières décennies, par l'émergence de mouvements indiens revendicatifs. C'est le cas dans le municipe de Huehuetla qui a vu l'Organisation indépendante totonaque (OIT), créée à la fin des années 1980 avec le soutien du clergé local, remporter les élections et gouverner le municipe pendant neuf ans (1990-1999). La lutte pour le pouvoir local, mais aussi le contexte national - avec le soulèvement du Chiapas - et international - avec les discours des organisations internationales sur les droits indigènes - ont contribué à mettre l'ethnicité au centre des enjeux du pouvoir local.

5 Le système religieux des Totonaques de Huehuetla peut être caractérisé par un culte des saints très dynamique dans lequel ont été élaborées des charges religieuses ${ }^{3}$. Dans le sud de la Sierra, le catholicisme est présent dans les pratiques cérémonielles : point de «compartimentalisation » religieuse apparente entre rituels païens et cérémonies catholiques comme c'est le cas chez les voisins septentrionaux, Totonaques " munixkan » (Ichon 1973, pp. 456-464), Otomi (Galinier 1997, pp. 55-57) ou Teenek (Ariel de Vidas 2002, p. 358 sqq.). Nous verrons que cette « compartimentalisation », pratiquement absente au niveau des rituels, est néanmoins présente dans la répartition symbolique des espaces.

6 D’après l'ouvrage dirigé par Lovell (1998, p. 13), dans différents contextes politiques et socio-économiques menaçants, des éléments cosmologiques peuvent être mobilisés et re-signifiés pour ériger certains aspects du " paysage » en support d'une définition de l'appartenance, permettant d'affirmer des "identités différenciées ». Cette pratique est illustrée par les Totonaques qui utilisent en effet la forêt - ou plutôt les espaces boisés en général - pour se créer une identité et appliquent la classification chaud/froid aux espaces écologiques. Ce principe dualiste, dont les origines à la fois mésoaméricaines et européennes ont été amplement discutées, notamment par Foster (1978) et López Austin (1996) ${ }^{4}$ est désormais très connu dans le domaine des pratiques thérapeutiques et culinaires, mais son application sur la répartition symbolique des espaces n'a pratiquement pas été étudiée dans l'aire mésoaméricaine.

\section{Une appropriation différenciée des espaces}

$7 \quad$ Huehuetla (État de Puebla), municipe à dominante totonaque (90 \% de la population), se situe au piémont de la Sierra Madre Orientale, sur la bordure méridionale de la Huastèque. Il s'agit d'une région fort accidentée, anciennement couverte d'une dense forêt subtropicale. Deux récoltes annuelles de maïs sont possibles grâce à une forte pluviométrie $\left(3000 \mathrm{~mm}\right.$ ) et une température moyenne de $22-24^{\circ} \mathrm{C}$. Le paysage est dominé, d'une part, par les caféières en sous-bois et la culture du maïs, toutes deux exploitées presque exclusivement par les paysans totonaques et, d'autre part, par l'élevage bovin des Métis.

8 La population (16 099 habitants en 2000) se répartit entre une bourgade chef-lieu, où se concentre la minorité 
métisse, et onze « villages » situés dans les montagnes. Ces villages, dans lesquels on parle la variante dialectale du totonaque dite « du sud de la Sierra » ou « de Gueytlalpa » (Stresser-Péan 1998), se caractérisent par un habitat semi-dispersé et une forte densité démographique (270 habitants par $\mathrm{km}^{2}$ pour l'ensemble du municipe). Le terme de "village » peut paraitre inadapté pour ces localités : leur "place centrale » ne regroupe qu'une dizaine de maisons autour de la chapelle, de l'école et de son terrain de basket. On pourrait être tenté de parler de " hameau », mais le terme est tout aussi inadéquat pour décrire ces communautés, appelées rancherías en espagnol et pulataman en totonaque (litt. " là où l'on vit »), puisqu'elles sont en réalité constituées de plusieurs centaines de maisons ou de huttes éparpillées sur les versants des collines et le plus souvent cachées sous le couvert forestier des caféières.

9 Dans le cadre d'un système de filiation patrilinéaire, l'habitat est organisé selon la règle de résidence patrivirilocale. Il en résulte des regroupements de maisons ou de huttes par « sections » qui réunissent frères et cousins d'une même lignée et sont appelées paks xtapuxnimat, soit « tous cousins ». En raison de l'héritage égalitaire entre les hommes, les terrains sont de plus en plus exigus : en général, ils correspondent tout juste à un jardin autour de la maison ou de la hutte et à la surface de la caféière (soit moins d'un hectare en moyenne). Dans certains villages, 60 à $80 \%$ des chefs de famille louent des terres pour cultiver le maïs, soit à d'autres chefs de familles totonaques du même village ou de municipes voisins, soit à des propriétaires métis résidant dans la bourgade.

Cette répartition différenciée de l'habitat entre Métis et Totonaques révèle une opposition sociale concentrique ${ }^{5}$. Les Métis, qui habitent la bourgade, conçoivent celle-ci comme un pôle de civilisation et de progrès ; ils cherchent à reproduire dans leur mode de vie les modèles urbains ou même étrangers. Ils se qualifient encore parfois selon l'expression coloniale de gente de razón ${ }^{6}$. À leurs yeux, les Totonaques, qu'ils désignent par les termes de inditos ou mozitos ou encore "gens des ranchos ", sont considérés comme des rustres ignorants qui vivent cachés dans les collines. L'expression "gente de rancho » se veut plus neutre que les autres, mais elle est tout aussi chargée sur le plan de la distinction identitaire par sa référence à l'espace. En miroir en quelque sorte, les Totonaques - lorsqu'ils s'expriment en castillan - désignent en général les Métis comme los del centro, « ceux du centre ». En langue vernaculaire, en revanche, les non-Indiens sont qualifiés de « serpents » ou luwanan (sing. luwan) en raison de leur propension, réelle ou supposée, à l'abus et à l'extorsion dans les relations commerciales, tendance souvent confirmée par l'observation empirique des relations économiques asymétriques entre paysans totonaques et commerçants-éleveurs métis.

11 L'expression spatiale de l'opposition socio-économique entre les deux groupes ne se limite pas aux formes de l'habitat. Les usages des ressources naturelles et les paysages qui en résultent sont aussi très contrastés.

\section{Agriculture intégrative vs. élevage extensif}

Du point de vue agro-écologique, le phénomène le plus marquant des dernières décennies est la disparition quasi totale de la forêt. Seuls quelques îlots au fond des ravins escarpés témoignent de la présence passée d'une forêt tropicale qui couvrait la majeure partie du territoire au début du Xxe siècle et qui représentait encore près de $40 \%$ de la superficie il y a seulement 40 ans (voir Figure 1). Plus qu'à la croissance démographique - et l'expansion de la 
frontière agricole qui en découle -, la disparition de la forêt est principalement due à l'essor de nouveaux modes d'exploitation des ressources naturelles, comme l'élevage bovin et la caféiculture commerciale qui ont été promus par les politiques de développement national (1970-1980). De cette évolution résulte aussi la sédentarisation des champs de maïs (milpas ou xawat) dont la culture itinérante reposait jadis sur l'existence d'une réserve écologique.

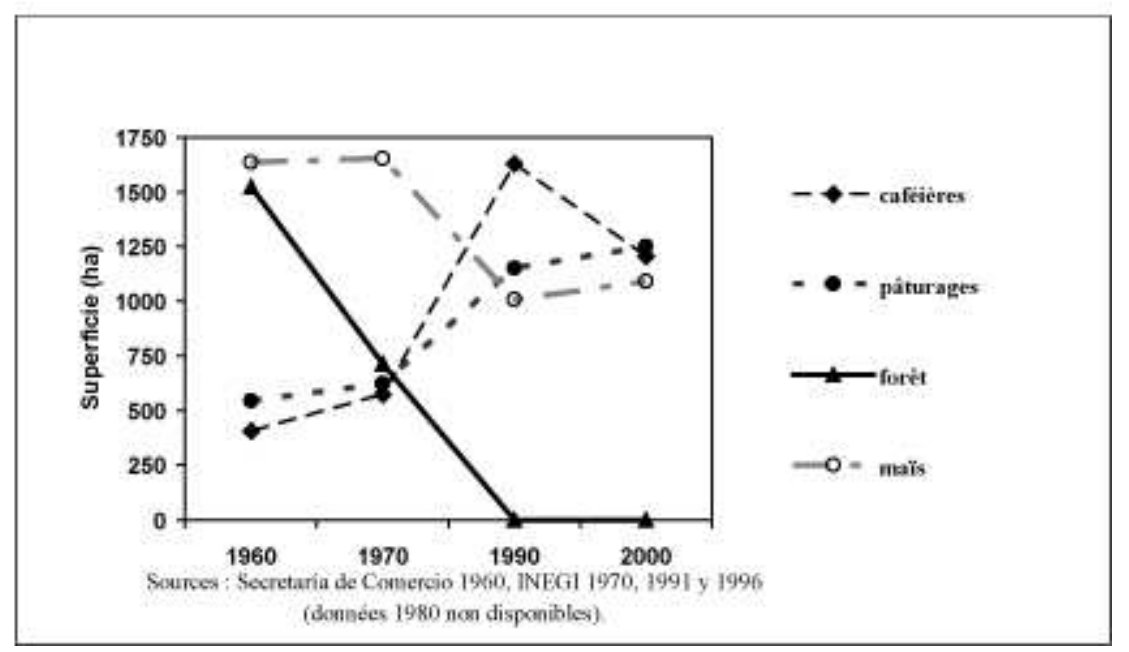

FIG. 1 - Graphique sur l'évolution de l'usage des sols en hectares, municipe de Huehuetla, 1960-2000.

\section{L'élevage}

Selon les recensements officiels, la superficie dédiée à l'élevage bovin a plus que doublé entre 1960 et 1990, passant de 524 à 1150 hectares. Cette expansion, notamment pendant les années 1970, s'est souvent réalisée grâce à l'achat et à la spoliation des terres des paysans totonaques par le biais de l'usure pratiquée par les commerçants du chef-lieu.

14 Sur l'ensemble du municipe de Huehuetla, les pâturages représentent actuellement plus de 30 \% de la surface agricole utile, pour seulement 44 exploitations avec en moyenne 26 hectares de pâtures par exploitation ${ }^{7}$. À l'opposé, la superficie restante - soit les $2 / 3$ du territoire - se répartit entre un peu plus de 2 ooo exploitations familiales, qui correspondent pour l'essentiel aux ranchos des paysans-caféiculteurs totonaques.

\section{La réaffirmation d'un usage agroforestier original : la caféiculture}

15 Dans la mosaïque des paysages agricoles de Huehuetla, en fort contraste avec les pâturages tout juste clairsemés de quelques arbres d'ombrage, l'enchevêtrement des milpas et des caféières en sous-bois est caractéristique des pratiques agricoles des paysans totonaques (Figure 2). L'agriculture de ces familles repose, d'une part, sur la 
production de maïs et de haricots, de fruits, de quelques légumes et herbes comestibles, d'autre part, sur la caféiculture sous couvert forestier. La production vivrière du champ de maïs, appelé xawat en totonaque, est presque exclusivement destinée à l'autoconsommation ${ }^{8}$; le café, lui, est pour la vente.

Ce dernier, initialement introduit par des commerçants-propriétaires métis à la fin du XIXe siècle (1880-1890), avait été adopté par les Totonaques dans leurs jardins sylvestres. Seulement quelques plants de café pourvoyaient à l'autoconsommation. La généralisation de la caféiculture, dans les villages totonaques pendant la période 1950-1960, se fit d'abord sous la forme d'une culture en sous-bois, le couvert forestier étant préservé. Les campagnes productivistes de l'Institut mexicain du café (INMECAFE), pendant les années 1970-1980, poussèrent ensuite à l'éclaircissement de l'ombrage, mais le modèle local d'agroforesterie résista à ces tentatives de « rationalisation » et se réaffirma après le démantèlement de l’INMECAFE en 1989.

Suivant les pratiques de culture en sous-bois fréquentes au Mexique et en Amérique centrale (Toledo et al. 1993, p. 84) ${ }^{9}$ la caféiculture des paysans totonaques de Huehuetla s'inscrit parfaitement dans un usage agroforestier diversifié des ressources et remplit plusieurs fonctions qui vont au-delà de la seule récolte de café pour la vente. Les plantations de café de Huehuetla font l'objet d'un traitement semblable à celui accordé à la forêt. La caféière (kakapejni en totonaque ou cafetal en espagnol), comme les bois (kakiwin en totonaque ou monte en espagnol), est une source de combustible pour le foyer, de bois de construction et de cueillette de nombreuses plantes utiles, qu'elles soient comestibles, médicinales ou à usage rituel et ornemental, sylvestres ou semi-domestiquées. C'est aussi un lieu pour la chasse aux oiseaux pratiquée par les garçons et d'intimité pour les jeunes couples.

18 Souvent, la caféière et l'espace immédiat autour de la maison ou de la hutte ne sont pas clairement séparés. Près de la demeure, soit dans un rayon d'une dizaine de mètres, il y a une sorte de jardin sylvestre où se concentrent certaines espèces cultivées ou semi-sylvestres consommées de manière courante - comme la chayotte maklhtukun (Sechium edule) ou les bananes sekgna (quatre variétés), le guaje (lilekg en totonaque, Leucaena pulverulenta) ou occasionnelle - comme l'avocatier kukuta, l'ananas akaxkat, le manioc kochkawi (Manihot esculenta). On trouve aussi des espèces employées pour l'alimentation de la basse-cour comme la "Trompette des Anges » (Brugmansia sanguinea).

19 Plusieurs plantes utiles, sylvestres ou semi-sylvestres, sont présentes dans la caféière : des herbes comestibles (quelites en espagnol local ou kaka en totonaque) et médicinales (tuwan) ; à l'étage arbustif, des espèces comestibles - comme, par exemple, un type de cardamome sylvestre (xkijit, soit Renealmia alpinia) - ou d'autres à usage ornemental et rituel - comme les balisiers xkatiyawat (Canna indica) ; à l'étage supérieur, des arbres fruitiers, sylvestres ou plantés - comme les sapotilliers (quatre espèces), le « poivre de Jamaïque » ukum (Pimienta dioica) - ou encore des espèces utilisées dans la construction - comme l'acajou cédrèle (Cedrela odorata). Soit en moyenne une trentaine d'espèces utiles, avec une variation de 25 à 50 espèces selon les caféières.

20 Par leur usage agroforestier, les caféières présentent des caractéristiques écologiques qui les rapprochent de la forêt "naturelle ", ce que confirment les études portant sur la production de matière sèche ou la diversité de l'avifaune (Jiménez et Gómez 1982). À la fois espace boisé « cultivé » et substitut de la forêt sur le plan des usages des ressources naturelles, cette forme de polyculture semi-sylvestre joue donc un rôle important, non seulement dans le maintien d'une importante superficie boisée ( $28 \%$ du municipe) mais aussi dans la conservation de plantes sylvestres utilisées par les familles totonaques. La caféière traditionnelle en sous-bois de Huehuetla peut donc être 
qualifiée d' « écosystème sylvestre cultivé ». Cet « agrosystème intégratif » (Garavaglia 1995, p. 1346) tranche donc avec l'usage mono-spécifique réservé aux pâturages. La classification des espaces, dérivée des principes recteurs de la conception totonaque du rapport au monde, permet de comprendre comment ces appropriations contrastées de l'environnement sont symboliquement mobilisées dans le contexte régional de confrontation interethnique.

\section{La hiérarchie dualiste des divinités tutélaires}

Les représentations totonaques de l'environnement, telles qu'elles sont exprimées dans le discours et les pratiques quotidiennes - notamment agricoles -, mettent en évidence la valeur opératoire d'une certaine vision du monde propre à la tradition mésoaméricaine. L'exposé de ces représentations cosmologiques et de la re-symbolisation des éléments catholiques dépasse largement le cadre de cet article. Il importe toutefois, pour l'analyse de la répartition symbolique des espaces, d'en évoquer les principes afin de comprendre comment ces représentations peuvent être re-signifiées dans le contexte contemporain de changements socio-économiques et de tensions interethniques.

22 La conception totonaque du monde est fondée sur le respect de la hiérarchie et de la réciprocité vis-à-vis des divinités tutélaires qui régissent les différents domaines du monde (katuxawat, litt. « là où il y a des champs de maïs »).

23 De manière semblable au schéma cosmologique des Totonaques du nord présenté par Alain Ichon (1969, p. 91), nous retrouvons une structure hiérarchisée des divinités principales et secondaires dans laquelle chaque espritmaître a la tutelle d'un domaine particulier de la nature et des activités humaines qui lui sont associées. En effet, il convient, pour éviter tout désagrément - accident, maladie, perte de l'âme -, de demander au xmalana, à l'espritmaître, l'autorisation pour toute intervention dans la sphère qui lui correspond : au maître de la forêt, Kiwi Kgolo', pour couper des arbres ; au maître de la terre, Xmalana Tiyat, pour semer ; au maitre des animaux pour chasser, et ainsi de suite.

La relation des humains avec les divinités est inscrite dans une logique de la reproduction des « cycles de la fertilité »- notamment celui du maïs et celui de la vie humaine - comme fondement de la sociabilité totonaque ${ }^{10}$. De même que les récoltes, l'harmonie sociale et la santé des hommes dépendent de l'accomplissement des devoirs rituels et des offrandes envers les divinités tutélaires, notamment vis-à-vis du saint patron Kinpuchinakan San Salvador. L'opposition nature-culture est étrangère à cette conception de type « analogique » qui repose sur «l'idée que les propriétés, les mouvements ou les modifications de structure de certaines entités du monde exercent une influence à distance sur la destinée des hommes ou sont elles-mêmes influencées par le comportement de ces derniers » (Descola 2001, p. 99). Dans la tradition mésoaméricaine, cette conception du rapport au monde et à autrui est caractérisée par l'idée d'une « co-essence » (López Austin 1997, p. 120) entre les humains, les plantes ou les animaux et les divinités, ainsi que l'illustre le complexe du nagualisme-tonalisme. À Huehuetla, l'association entre une personne et un double animal, bien que connue ${ }^{11}$, n'est plus en usage. Dans la région, la co-essence entre humains et non-humains trouve son expression dans la logique totonaque de classification nosologique et botanique selon les polarités du chaud et du froid. Cela apparaît par exemple dans le fait que, pour les Totonaques 
de Huehuetla, le maïs et les humains partagent un même principe vital de qualité « chaude », appelé listakni', que l'on peut traduire littéralement par « ce qui fait grandir, pousser ». Cette co-essence implique que hommes et plantes sont protégés par les mêmes remèdes utilisés contre certaines influences néfastes et, donc, considérées comme «froides » (une éclipse, le « mauvais souffle » d'un mort, etc.). Lors de l'une de mes participations aux semailles, le propriétaire du champ avait mélangé aux grains de maïs des feuilles de tokgxiwa (Sambucus mexicana ou sauco en espagnol), plante de qualité chaude utilisée pour guérir une personne qui a perdu son âme. Cette précaution était nécessaire pour protéger l'âme du maïs de l'influence néfaste d'un mort car l'un des aides avait participé la veille à un enterrement, ce qui risquait de faire pourrir le semis.

\section{La divinité solaire San Salvador}

Le rôle que les Totonaques de Huehuetla attribuent au saint patron Kimpuchinakan San Salvador - c'est-à-dire Jésus Saint-Sauveur - met en relation le Christ avec le soleil et le maïs. San Salvador n'est en effet pas seulement le saint éponyme de Huehuetla. Il se confond avec Dieu, Kimpuchinakan, dont il est en quelque sorte la manifestation locale, et est associé au soleil - la couronne iridescente de saint Sauveur en est le symbole - et à la croissance des plantes cultivées - notamment le maïs et le piment. Selon une adaptation chrétienne de la tradition mésoaméricaine, les principaux cultigènes sont considérés comme ayant été engendrés par des gouttes de sang divin tombées sur la terre lors de la crucifixion du Christ ${ }^{12}$.

La connexion du saint patron avec les semences et la fertilité est clairement illustrée dans de nombreux récits sur l'esprit de saint Sauveur délaissant Huehuetla en raison d'une offense. L'image de saint Sauveur, jalousement gardée sur l'autel principal de l'église du chef-lieu, est bien une "réplique » dans le sens attribué à ce terme par López Austin (1997, p. 145) : il existe une « co-essence » entre l'image (ou l'idole) et la divinité qu'elle représente. Selon les informateurs, elle est en effet investie par l'esprit de Kimpuchinakan et, lorsque les précautions rituelles liées à son culte ne sont pas respectées, l'esprit de la divinité - listakni’ ou xnaku, « son cœur » - délaisse l’image. Afin de prévenir ce risque, il faut honorer le saint lors des fêtes, appelées mayordomías, au cours desquelles plusieurs chefs de famille du village organisent chacun un banquet, une procession et font des offrandes - notamment des cierges décorés - en l'honneur du saint patron. Si, courroucé en raison d'un manque de respect, l'esprit du saint abandonne l'image et part de la communauté, il emporte avec lui « ses semences », ce qui affecte directement la fertilité du maïs et des piments ou se manifeste par une sécheresse ou des pluies excessives qui sont tout aussi néfastes pour les récoltes.

27 De nombreux témoignages ${ }^{13}$ font référence à des offenses faites à l'encontre du saint patron pour expliquer telle année de sécheresse ou telle mauvaise récolte. La dernière en date a été concomitante à un changement dans l'autel principal, voulu par le curé contre l'avis des sacristains (fiscales) et, donc, considéré par eux comme étant la cause des pluies torrentielles qui firent pourrir le maïs. On peut reconnaître là une logique complémentaire à celle de la « disparition des semences » : celles-ci germent bien, mais ne sont plus protégées contre les effets néfastes de la pluie torrentielle. Selon les explications livrées par les informateurs, la pluie est bien sûr nécessaire et bénéfique, mais elle est la manifestation d'une force complémentaire à la force solaire de saint Sauveur, néfaste lorsqu'elle 
n'est pas compensée par celle du Christ soleil. Ce pouvoir, opposé mais complémentaire à celui du Christ soleil, est aussi celui des divinités de la pluie et de l'eau, San Miguel et San Juan Aktsini'. On voit déjà se dessiner une opposition cosmologique dans les forces divines entre celles qui sont « chaudes » et celles dites " froides », opposition classique dans la cosmologie mésoaméricaine (Galinier 1997 ; López Austin 1996, 1997).

\section{Les dieux du tonnerre et de la pluie}

San Juan Aktsini' est la divinité de la tourmente associée aux tonnerres jilinin, faiseurs de pluie, qui correspondent, pour les Totonaques septentrionaux, aux quatre piliers du monde (Ichon 1969, p. 124) ${ }^{14}$. Il est connu pour vivre au fond de la mer où il attend qu'on lui annonce sa fête (le 24 juin) pour provoquer un déluge. Une nuit, au début du mois de juin, une tourmente avec des éclairs suscita, chez mon hôte Manuel, les commentaires suivants : « Juan commence à sortir de la mer, il veut qu'on lui fasse sa fête, il veut ses "poulets", c'est-à-dire ses chrétiens ». En effet, responsable des noyades, San Juan Aktsini' est avide d'âmes humaines (les poulets sacrificiels sont une métaphore de la chair humaine). Les âmes des morts noyés l'accompagnent et sont ses aides. Il en est de même pour le génie des sources et des rivières, le maître de l'eau, Xmalana Chuchut, qui peut causer une perte de l'âme ou un « effroi » (tapekwan ou susto en espagnol), notamment chez les petits enfants lorsqu'ils tombent dans l'eau.

29 Ces croyances autour de San Juan Aktsini' renvoient au complexe mésoaméricain du domaine de l'eau et des âmes des noyés, le Tlalocan, qui est aussi la montagne sacrée des richesses où sont générées les semences et les forces fertiles ${ }^{15}$. Les attributs attachés uniquement à San Juan (ou saint Jean) dans le nord sont répartis, dans le sud de la Sierra, entre lui et San Mikilh (San Miguel ou saint Michel). Ce dernier prend de l'ascendant sur saint Jean dans la hiérarchie divine. C'est non seulement le cas à Huehuetla, mais aussi dans les municipes voisins, notamment à Zozocolco où San Miguel est le saint patron éponyme et revêt un caractère solaire équivalent à celui de San Salvador à Huehuetla. La figure du saint patron de Zozocolco est profondément dualiste, car il est aussi le maître de l'éclair, de la pluie et des sources ${ }^{16}$. 


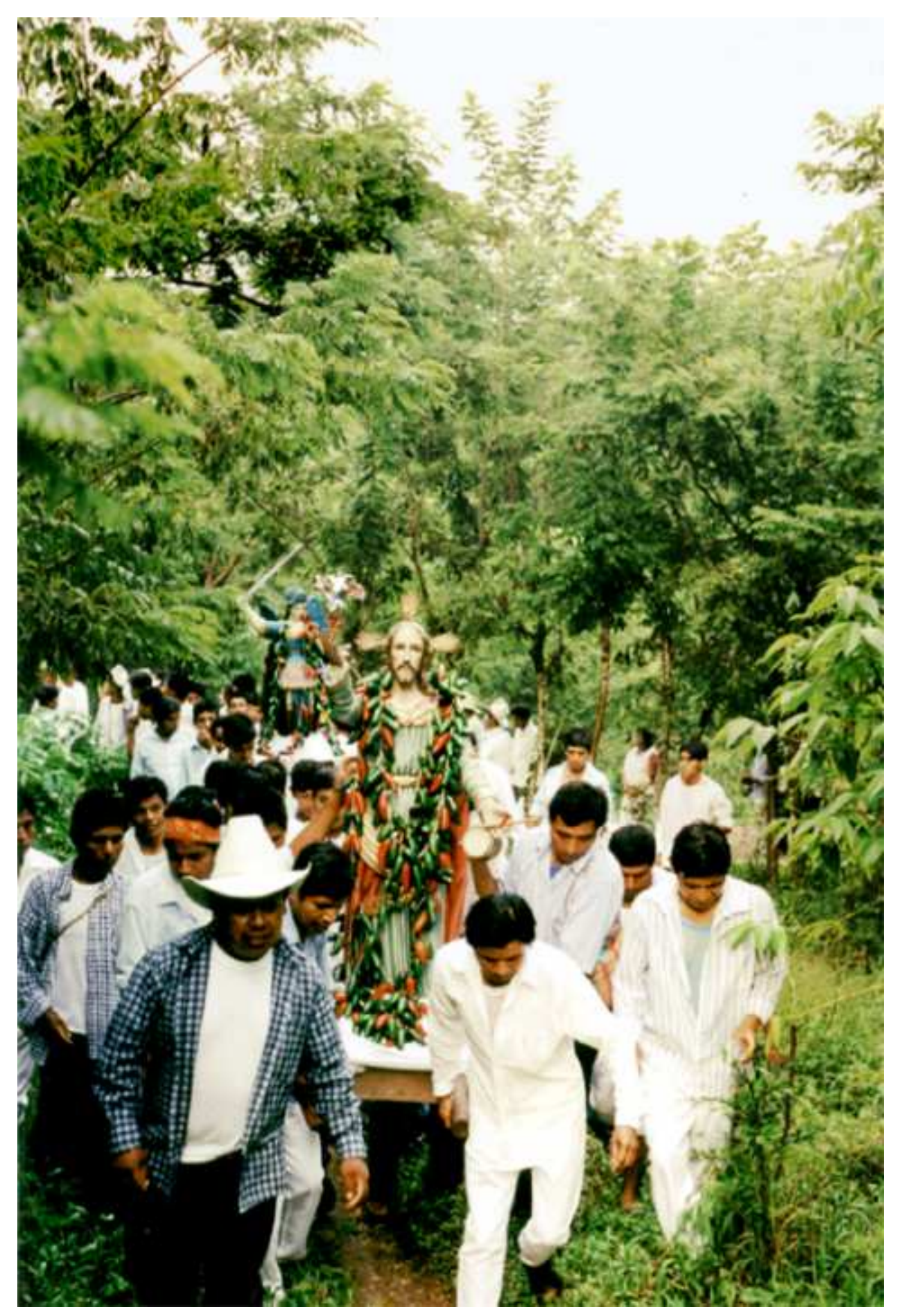


FIG. 3 - Les images de San Salvador et de San Miguel en procession lors de la fête patronale de Huehuetla (cliché Ellison).

À Huehuetla, dans la procession qui est organisée pour la fête patronale, saint Michel partage la place d'honneur avec le Dieu patron, San Salvador (Figure 3). En totonaque, on l'appelle Kinpuchinakan Mikilh, " notre Dieu Miguel » ou " notre patron Miguel ». Ce que les habitants de la région expliquent de la manière suivante : « nachuna xpuxko kinkachikinkan », c'est-à-dire « il est aussi le chef, le patron de la communauté ». Il occupe donc une place hiérarchique proche - pratiquement équivalente - de celle du Christ soleil (San Salvador). Le rôle bienfaiteur du dieu de la pluie unique du nord de la Sierra (Aktsini') est ici réservé à saint Michel, dieu de la pluie bénéfique, distinct de San Juan Aktsini'. Ce dernier conserve les caractéristiques les plus néfastes en tant que dieu de la tempête et responsable des morts par noyade. Tandis que saint Michel est principalement associé à la pluie, ou « l'eau d'en haut », saint Jean ou San Juan Aktsini' renvoie aux rivières, c'est-à-dire « l'eau d'en bas ». L'axe classificatoire chaud/froid est donc ici dédoublé par l'axe haut/bas. Selon le premier axe, San Juan est parfois confondu avec un être chtonien associé aux espaces sylvestres, le maître de la forêt appelé Kiwi Kgolo' (litt. " arbre vieux »).

\section{Kiwi Kgolo', maître de la forêt}

Il s'agit d'un esprit ou d'une divinité secondaire reliée au domaine humide et froid sous la tutelle de San Juan Aktsini'. Il se manifeste généralement de façon sonore, la nuit ou les jours de brume, donc quand le soleil n'est pas visible. On entend des coups de hache ou de machette sur les arbres, mais on ne voit personne et aucun arbre abattu n'est retrouvé. Il va sur les sentiers peu fréquentés, prend l'apparence d'une personne familière et enchante celui qui s'aventure dans les bois. Il invite sa victime dans sa " maison » et l'enferme dans un aven (musink) où il lui donne des champignons en disant : "Tiens, mange du pain » ${ }^{17}$. Kiwi Kgolo’ et Xmalana Takgalhinin, le maitre des animaux, sont parfois confondus. Le second désoriente le chasseur excessif et l'amène dans une grotte où les animaux sauvages sont parqués ${ }^{18}$. Le maître de la forêt peut aussi prendre l'aspect d'un homme-arbre pour effrayer ceux qui essaieraient de couper un arbre sans lui avoir fait l'offrande d'eau de vie. Kiwi Kgolo' joue donc un rôle semblable à celui des chaneques parmi les Nahua du Veracruz (Blanco et al. 1992) : il viendra punir ceux qui coupent de manière immodérée ses arbres en leur infligeant un effroi - tapekwan - et les complications de santé qui en découlent.

Dans la version du mythe des jumeaux du maïs recueillie par Münch (1992) auprès des Totonaques des basses terres, Kiwi Kgolo' est « l'arbre des profondeurs de la jungle, le seigneur de la forêt », " ami protecteur » de celui des deux jumeaux qui se transformera en soleil. C'est suivant les instructions de Kiwi Kgolo' que le jeune dieu « donna l'ordre aux animaux et aux arbres de l'aider à sauver nos ancêtres de la grande obscurité et faire naître les nouvelles générations » en semant le maïs (ibid., p. 289, traduction de l'auteur, N. E.). Or, dans une version présentée par Ichon (1973, p. 125), c'est saint Jean (ou Aksanjan) qui essaie de cultiver le maïs avec l'aide des animaux de la forêt dont il est le maître, mais cette situation contrevenant à l'ordre cosmique - le Soleil étant le véritable maître du maïs - est vouée à l'échec. Il ne fera pousser que des tubercules. Comme le souligne López Austin (1997, p. 141), « pour les Totonaques, chacune des forces cosmiques est régie par son gouvernant » : alors 
que le Christ soleil gouverne la partie chaude et sèche du monde, San Juan commande la partie froide et humide. Ainsi le sang du soleil (ou du Christ) a donné naissance aux cultigènes qui poussent en surface à la chaleur de ses rayons, tandis que saint Jean est à l'origine des tubercules et tout ce qui pousse sous terre grâce à la pluie (Ichon 1973, p. 126).

33 Aksanjan pour le nord de la Sierra totonaque et Kiwi Kgolo' pour le sud correspondent au même esprit maître de la forêt. D'ailleurs, à Huehuetla, Kiwi Kgolo' est aussi appelé Xwan Xalak Kakiwin ou Juan del Monte (Jean de la Forêt), comme son corollaire septentrional. Sans se confondre avec San Juan Aktsini', maitre de la tourmente et des rivières ${ }^{19}$, Jean de la Forêt, Kiwi Kgolo', s'inscrit bien dans le complexe de la montagne sacrée et de l'inframonde, lequel fait écho au «paradis de brume » du monde de la fertilité, le Tlalocan des anciens Nahua et que ceux de Cuetzalan, proches des Totonaques, continuent à appeler Talokan (Aramoni 1990).

\section{Le symbolisme sylvestre dans le schéma dualiste}

L'organisation dualiste du panthéon totonaque, déjà décrite par Ichon (1973, pp. 35-50) qui insistait sur la répartition symbolique des divinités selon les axes haut/bas et sud-est/nord-ouest, est renforcée par la classification des domaines de « compétence » des saints et des « maîtres » selon l'axe chaud-sec/froid-humide.

35 Le calendrier des saints célébrés par les villages du municipe de Huehuetla et les deux cycles du maïs, l'un correspondant à la moitié « chaude » et « sèche » de l'année, l'autre à la moitié «froide » et « humide », mettent en évidence la relation qui existe entre le culte des principaux saints et les étapes initiales et terminales de ces cycles agraires reproduisant dans l'organisation temporelle la logique de la répartition dualiste des divinités selon l'axe chaud/froid (Ellison 2004, pp. 349-352).

36 Mais la répartition des rôles des divinités ne s'inscrit pas seulement dans une " géographie mythique »: les domaines de tutelle des saints et des esprits-maîtres correspondent aux lieux des pratiques quotidiennes, notamment agricoles et thérapeutiques ; c'est évidemment le cas des bois qui sont sous la tutelle de Kiwi Kgolo', mais aussi des champs de maïs associés au saint patron San Salvador ou, encore, de l'espace domestique lié aux saints catholiques en général. Par ailleurs, comme dans l'ensemble de l'aire mésoaméricaine et même bien au-delà, la valeur opératoire du principe classificateur de la polarité chaud/froid est manifeste dans les pratiques thérapeutiques et la classification indigène des plantes. Les informateurs expliquent les qualités « chaude » (chichí) ou « froide » (skga'wiwi) ${ }^{20}$ d'une plante selon au moins trois critères qui peuvent se recouper : la teneur en eau de la plante, son usage thérapeutique et son biotope. Ainsi, une plante qui pousse dans la forêt est souvent considérée comme « froide » - c'est le cas, par exemple, de Solanum nudum (ou akgtsitsipuskat) utilisée contre le « mauvais souffle » ( « un » en totonaque ou « mal aire » en espagnol local). Une autre sera dite " chaude » parce qu'elle pousse en des endroits ensoleillés ou pierreux : l'arbre puklhnan kiwi (Croton draco), à la sève cicatrisante, est chaud " parce qu'il pousse au soleil et dans les pâturages ». Ce critère, fondé sur les caractéristiques écologiques et édaphiques, indique que les catégories de « chaud » et de « froid » peuvent aussi s'appliquer aux biotopes ${ }^{21}$. Ces critères mobilisés par les Totonaques dans la classification des plantes sont inséparables des représentations symboliques des espaces écologiques. 
À ce titre, si la forêt, kakiwin (litt. « là où il y a des arbres »), est perçue comme un espace « froid », cela ne s'explique pas seulement par la fraîcheur du couvert forestier. En fait, elle renvoie également à l'inframonde, froid et humide, au monde sauvage de la «montagne » et aux esprits chtoniens qui vivent dans les grottes : le maitre de la forêt (Kiwi Kgolo'), le maître des animaux (Xmalana Takgalhinin) et leurs aides. Tout comme les Baatsik' chez les Teenek veracruzains (Ariel de Vidas 2002, pp. 174-177), ces esprits se confondent avec les ancêtres antédiluviens qui se cachèrent du soleil en s'enfouissant sous terre et donnent à ces lieux sombres - les grottes, les ravins, la forêt - un aspect dangereux pour l'homme qui ne respecterait pas les règles de réciprocité dans le rapport au monde sylvestre, notamment lors de la coupe d'arbres ou de la chasse.

38 Entre la forêt et les cavernes s'établit un rapport de continuité symbolique ${ }^{22}$. La forêt était pendant longtemps le « contenant » des grottes et, aujourd'hui encore, grottes et bois résiduels se situent souvent au fond des gorges et ravins. Mais, plus que par cette association spatiale, c'est au niveau de la classification commune dans un domaine régi par Kiwi Kgolo' (maître de la forêt) et le Xmalana Takgalhinin (maître des animaux) que s'établit cette continuité. En effet, comme nous l'avons déjà mentionné, ces deux maitres du monde sylvestre, étroitement liés entre eux, parfois jusqu'à se confondre, ont leurs demeures dans les entrailles des montagnes (Kiwi Kgolo' conduit ses captifs dans le musink, l'aven ; le Xmalana Takgalhinin, par l'intermédiaire de ses animaux, entraîne le chasseur excessif dans une grotte).

Dans la tradition cosmologique mésoaméricaine, de même que dans celle de Huehuetla, les cavernes ont une signification particulière : ce sont des lieux privilégiés de communication avec l'inframonde - et avec ses forces bénéfiques (fertilité) et néfastes (les « mauvais souffles ») -, avec les ancêtres aussi, ceux de la première humanité, qui se sont enfouis sous les montagnes à l'apparition du soleil (ou du Christ). Au-delà de cette tradition orale, une part de cette symbolique relative aux grottes a été conservée dans certaines pratiques religieuses des Totonaques de la région de Huehuetla. Des grottes sont réservées au culte de la fertilité, d'autres aux pratiques chamaniques (qualifiées de sorcellerie, brujería). Avec la disparition de la forêt, les ravins escarpés et les grottes semblent aujourd'hui être les ultimes réduits des espaces «sauvages ». Les Totonaques entretiennent avec les esprits qui habitent ces lieux une relation de crainte car ils les considèrent comme étant dotés de pouvoirs dangereux. Parmi ces esprits, on retrouve donc Kiwi Kgolo' dans la forêt et le maître des animaux dans les grottes, mais il y a aussi des serpents dans les grottes et les cascades, des esprits jaguars dans les rochers des ravins, etc. C'est pour gagner leur bienveillance que les Totonaques font preuve de respect à leur égard.

40 La continuité symbolique qui est donc établie, par la tradition totonaque, entre la forêt et les grottes range la première du côté froid-humide dans l'ordre cosmologique. Dans quelle mesure les autres espaces boisés sont-ils explicitement classifiés en ces termes ? Quelles sont les implications des profonds changements agro-écologiques des dernières décennies sur ces représentations de l'environnement?

\section{Les arbres comme référents symboliques dans le rapport aux métis}




\section{Le kakapejni (jardin de café), refuge du symbolisme sylvestre ?}

41 Comme nous l'avons noté plus haut, la caféière de Huehuetla (kakapejni ou cafetal) possède les caractéristiques d'un système agroforestier. Cette forme d'association de cultures semi-sylvestres, ou de " forêt anthropogénique ", joue un rôle primordial dans la conservation d'une importante superficie boisée et des pratiques d'usage des ressources sylvestres, alors que la forêt à proprement parler se limite désormais aux fonds de ravins. Ce profond changement écologique, à travers lequel la forêt disparaît, mais où le kakapejni perpétue en partie les fonctions écologiques de celle-ci, pose un problème classificatoire du point de vue des représentations de l'environnement : à quelle catégorie rattacher cet espace ? à celle des espaces cultivés ou à celle de l'espace sauvage de la forêt ? Le kakapejni remplace-t-il aussi la forêt sur le plan symbolique?

42 À cette dernière question, trois éléments nous incitent à répondre par l'affirmative. Le premier réside dans le caractère polysémique du mot kakiwin, littéralement « lieu des arbres ». Ce terme, tout en se référant plus spécifiquement à la forêt non cultivée, s'applique aussi à la « campagne » en général (la forêt ayant été le contenant autant de la milpa que des espaces habités). Il s'applique même parfois directement aux caféières : on peut dire indifféremment « je vais dans ma forêt » (kama kinkakiwin) ou « je vais dans mon cafetal » (kama kinkakapejni). Cette dénomination sous le même vocable de la forêt et de la caféière existe aussi dans d'autres groupes indiens producteurs de café (Alcorn 1984, p. 372 ; Katz 2000, p. 174).

43 Néanmoins, le kakapejni se distingue de la forêt, car il s'agit d'un espace travaillé par l'homme, ce qui le rapproche des espaces agricoles. Comme pour d'autres cultures, le café a son maitre, ou xmalana, qu'il s'agisse de saint Sauveur (San Salvador) pour le café rustique, dit « créole », spécifiquement ou de la Vierge de Guadalupe pour le travail du café en général. Lors de la procession pendant la fête de cette Vierge (le 12 décembre), les fiscales - les gardiens des images sacrées dans l'église du chef-lieu - l'ornent d'un collier de cerises de café, synonyme de prospérité ; celui-ci fait écho à l'image de San Salvador qui porte un collier de piments en signe de fertilité lors de la fête patronale en août. La plante de café a donc été intégrée aux pratiques rituelles. Si la caféière est rattachée à la forêt dans la classification des espaces, les représentations du café en tant que plante cultivée se rapportent au symbolisme des plantes domestiquées associées aux saints catholiques. En ce sens la caféière occupe une position intermédiaire dans la classification des espaces. Le second élément qui permet d'inclure la caféière dans les représentations de la forêt va de pair avec la croyance, encore actuelle, selon laquelle le maître de la forêt, Kiwi Kgolo', n'est pas confiné aux espaces « sauvages » que sont les ravins, il apparaît aussi dans les plantations de café sous futaie. Le troisième élément renvoie à la place de la caféière aux côtés de la forêt dans la classification totonaque des espaces écologiques selon l'axe « chaud/froid».

44 La reproduction de ce principe dualiste dans la classification des espaces écologiques montre comment les Totonaques mobilisent les représentations qu'ils se font des espaces boisés dans la construction d'une identité différenciée par rapport au groupe dominant que sont les Métis et comment ils étendent ce symbolisme sylvestre à la caféiculture en sous-bois.

« Nous, nous vivons parmi les arbres, parmi les feuillages » 
Les commentaires non enregistrés, issus de conversations informelles in situ lors des travaux agricoles et ceux suscités par des photographies de la région ${ }^{23}$, nous ont beaucoup appris sur la perception et la conception totonaque de l'environnement dans le contexte socio-économique actuel. Ils expriment clairement les oppositions entre les espaces indigènes et les espaces métis, autant par les contrastes flagrants dans l'habitat que dans l'appréhension du paysage. Or, en tenant compte des critères de classification des plantes énoncés plus haut, les extraits présentés plus loin montrent que les Totonaques traduisent ces contrastes en termes d'opposition chaud/froid en fonction de la présence ou non d'arbres dans le paysage.

Une informatrice (50 ans) décrit ainsi les alentours de Lipuntahuaca (Figure 2) :

1. Uyma wi kakiwin, wilakgolh sipi, 2. wilakgokan nata niku tlakg yan kiwi yakgon chiki ni tasiyu. [...] 3. Uynu k' San Rafael xalakaputu xa kilhtutu puxkga uyma wi kachikin. 4. Anta win tapaxuwan kakiwi lamaw akinin.

1. Là, c'est le monte [la forêt, la campagne], il y a des collines, 2. là où il y a beaucoup d'arbres, il y a des maisons qu'on ne voit pas. [...]. 3. Là c'est San Rafael, là où les trois ravins se réunissent, c'est un village. 4. Ici dans la forêt, nous vivons avec joie.

L'absence d'arbres là où habitent les Métis est encore soulignée dans plusieurs commentaires faits à propos d'un cliché pris dans le « centre » du chef-lieu (Figure 4):

1. Tasiyu xkachikin anta puitat nitu xan tuwan porque uyma tani wilakgo' luwanan ni an xlakgaskinkgoy xlan kiwi, 2. Lakaski magolh xatlan xa x'chik'kan. 3. Uyma tasiyu kachikin, anta kachikin, 'tak kinkachikinkan

Huehuetla, 4. ukxilhmaw lantla xla wi tapaxuwan wi anta k'chik, ni akgatli x anta lipuwan, porque tlan xan tasiyu x'chik 5. pus ay takaxtlawnit taniku xla wi xputawilh.

1. Ici on voit le bourg, dans le « centre », il n’y a pas de végétation [litt. « pas de feuilles »], parce que les Métis n'ont pas besoin d'arbres, 2. eux ce dont ils ont besoin c'est d'avoir une belle maison. 3. Là on voit le bourg, dans le centre du chef-lieu, dans notre village de Huehuetla, 4. nous voyons comment ils vivent heureux dans leurs maisons, ils n'ont pas de problèmes 5 . car ils ont de tout, il ne leur manque rien là où ils habitent. 


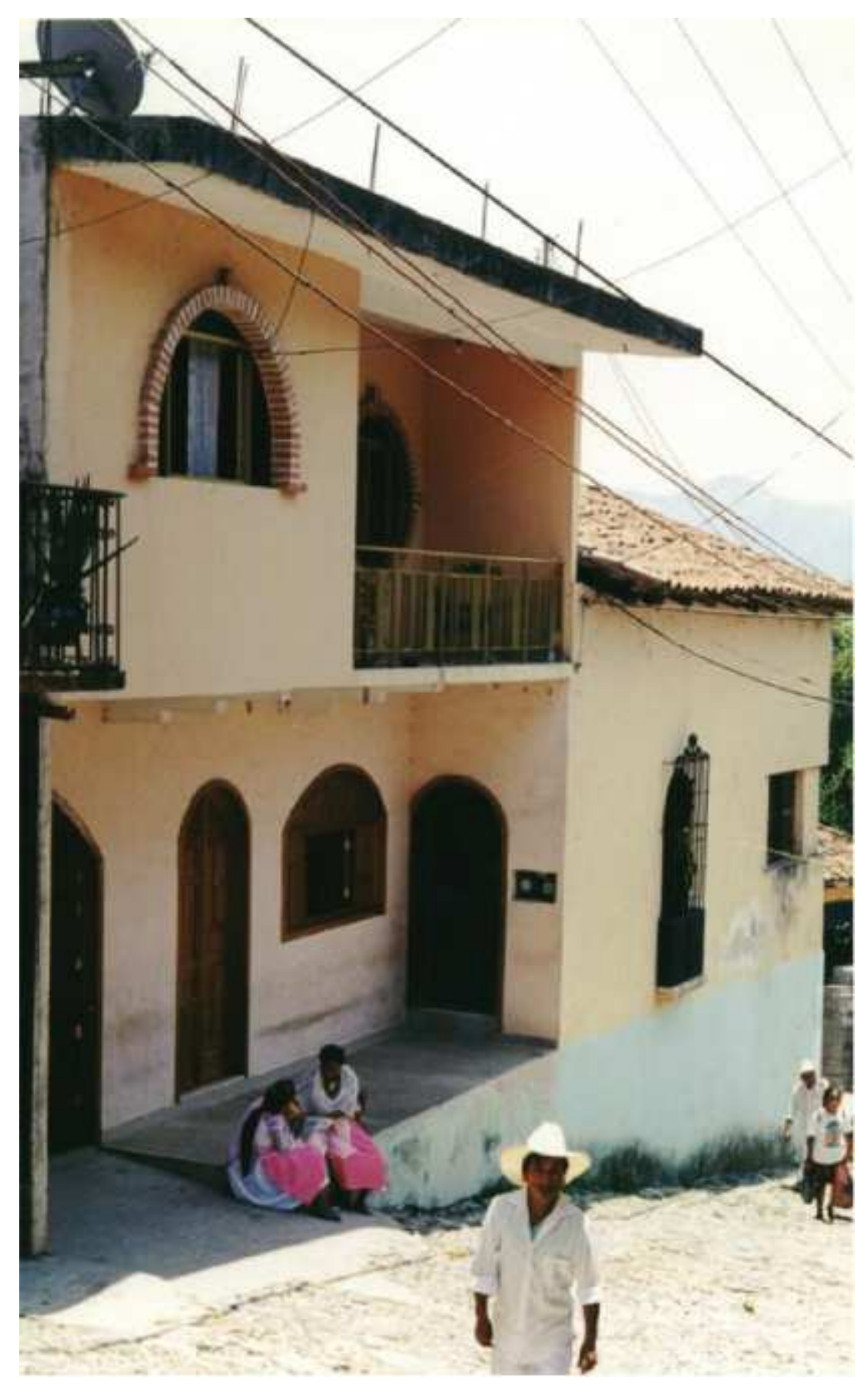

FIG. 4 - Maison d'un éleveur de bétail, bourgade de Huehuetla (cliché Ellison). 
Renforçant l'identification de l'habitat et de la vie totonaque avec les espaces boisés, le commentaire d'une photographie montrant sur une même pente des pâturages, des champs de maïs et des jardins de café (Figure 5) insiste sur le fait que, par opposition aux pâturages, pour travailler la milpa les paysans laissent des arbres :

1. Tasiyu kgastin, hasta tokanacha, tasiyu tanin taskujnit, 2. tasiyu tanin kgastin lamakogkan anta uymak Leakaman, 3. uymak Leakaman anta uyma tasiyu xkilhpan potrero 4. tasiyu yakgolh laktlanka kiwi, tani makgstakgkanit naktlanka kiwi tapaxuwan tani wi katiyatni, skga'wiwi wi ntiyat porque yakgolh kiwi. 5. Taniku k potrero, pus, luka chichi tasiyu, lipekua chichinan.

1. On voit les collines, on voit où les gens ont travaillé sur la colline, 2 . on voit la colline où habitent les gens de Leakaman, 3. sur une partie de la colline de Leakaman, on voit un pâturage 4. là où on travaille la terre, on voit qu'il y a de grands arbres, là on voit la joie de la terre, la terre est fraîche parce qu'il y a des arbres. 5 . Là où il y a le pâturage, on voit qu'il y a beaucoup de soleil, il fait très chaud. 


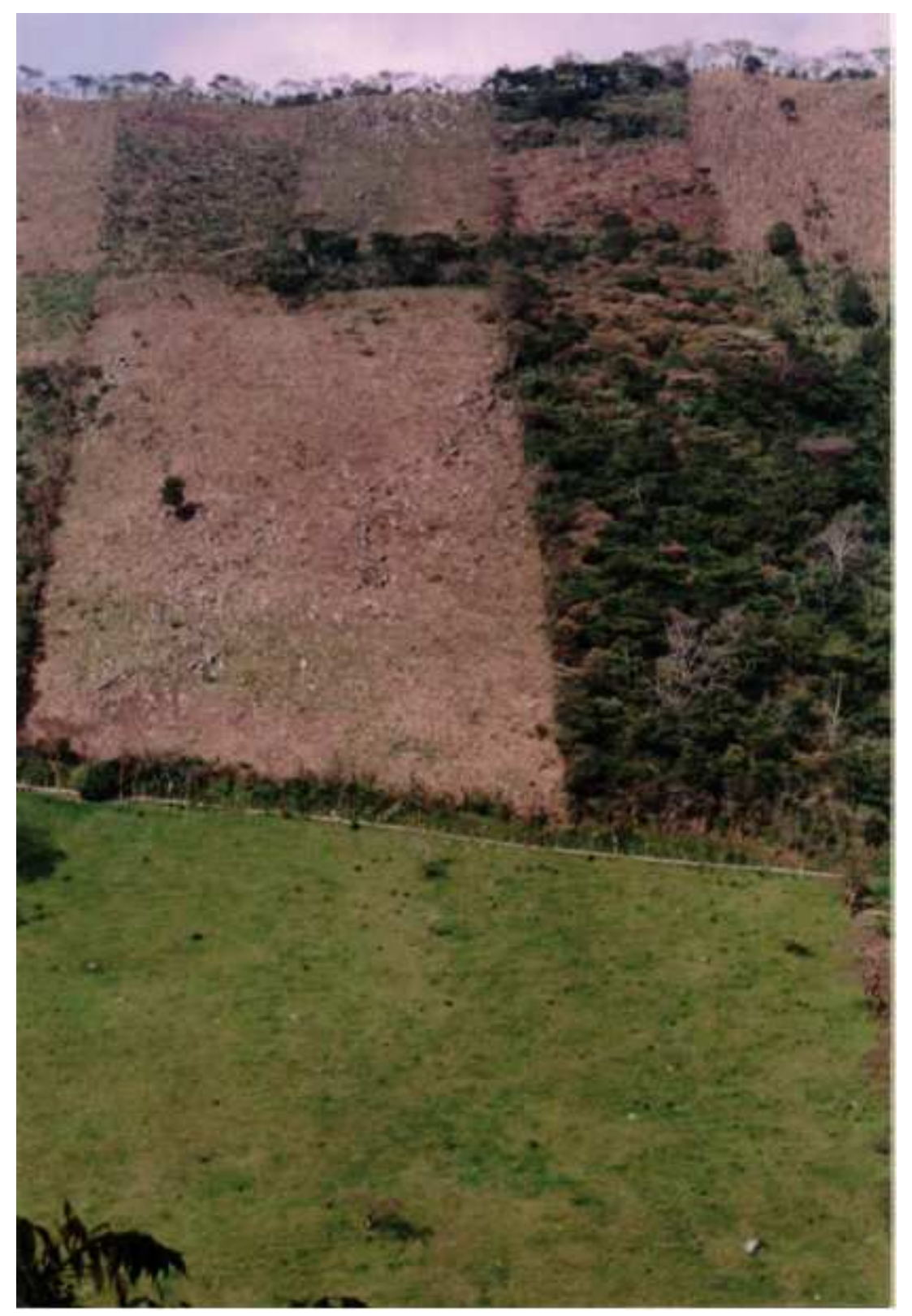

FIG. 5 - Pâturage (en bas), milpas fraîchement sarclées et caféière, Huehuetla-Leakaman (cliché Ellison).

L'informatrice conclut par ce commentaire significatif : «akinin lamaw kakiwinin, akinin xalnin katuwanin », soit : « Nous, nous vivons dans la forêt, parmi les feuillages ».

50 Mais, ce dernier extrait laisse entrevoir un nouvel élément qui permet de décrire les terres travaillées par les Totonaques et de les apprécier en contraste avec les pâturages appartenant aux Métis : l'opposition chaud/froid ou 
frais. Tout comme le premier extrait expliquait la joie de vivre en des lieux boisés, celui-ci décrit la « joie de la terre » rafraîchie par les arbres que les paysans totonaques laissent pour cultiver leur café, par opposition au pâturage dépourvu de couvert forestier et où la terre a chaud. Le même principe de polarité qui prévaut dans la classification des plantes en fonction de la qualité « chaude » ou « froide » attribuée aux biotopes est donc appliqué aux espaces écologiques dans les descriptions de paysages.

51 Les thèmes prédominants pour décrire un paysage varient en fonction des clichés photographiques. Pour les personnes interrogées, le critère de l'appartenance ethnique semble permettre de distinguer deux groupes. Dans le premier (qui comprend les clichés des hameaux totonaques sur lesquels les pâturages - ou, plus précisément, des manifestations évidentes de la présence métisse - sont absents), les commentaires portent sur la végétation, la fertilité, la présence de fleurs, la vie heureuse dans un paysage qui est perçu comme beau dans son ensemble, mais aussi comme éventuellement dangereux lorsqu'il s'agit plus spécifiquement d'un espace sauvage (ravins, grottes). L'aspect esthétique est bien mis en avant dans les expressions utilisées : stlan tasiyu, « c'est joli à voir ». Cependant cette appréciation n'est pas fondée sur une dimension artistique, mais sur des référents cosmologiques ${ }^{24}$. Ceux-ci renvoient soit au travail agricole perçu comme un accompagnement des cycles de fertilité qui sont régis par les divinités tutélaires, soit aux espaces boisés conçus comme une manifestation de la fertilité de la terre, habitée par les esprits chtoniens des ancêtres. La vivacité de cette " part idéelle » dans les pratiques agricoles conditionne sans doute cette extension du dualisme chaud/froid à la perception des espaces agro-écologiques. Notons que ce discours valorisant l'environnement indigène est présent parmi d'autres minorités revendicatives ou " fières » - par exemple les Triquis du Oaxaca (Katz 1991, p. 28) ; en revanche, les groupes qui élaborent un discours identitaire fondé sur l'autodénigrement - comme c'est le cas chez les Teenek veracruzains (Ariel de Vidas 2002) ou chez les Mixtèques (Katz, ibid.) - parlent de leur environnement en des termes évoquant la laideur.

52 Pour ce qui est du second groupe de clichés (qui montrent des pâturages ou le bourg chef-lieu, donc des photographies sur lesquelles la présence des Métis apparaît clairement), les descriptions relèvent du registre socioéconomique (« ils vivent dans la gloire ») ou de contrastes écologiques (« eux, ils n'ont pas besoin d'arbres », alors que « là où travaillent nos frères, il y a de grands arbres, on voit la joie, la terre »). Les Totonaques emploient souvent le symbole de l'arbre, notamment de l'acajou « cédrèle » (puksnankiwi : Cedrela odorata), comme une métaphore du pouvoir dans le cadre de la rhétorique de différents projets (coopératives, organisations politiques, projets de l'INI). Les promoteurs de l'Organisation indépendante totonaque - mouvement dont les revendications ethnico-écologiques trouvent un écho favorable dans tous les villages de Huehuetla - comparent, par exemple, leur organisation et la communauté de Huehuetla à un cedro : " les racines, ce sont nos traditions, le tronc c'est la communauté ( el pueblo » ou chuchutsipi ${ }^{25}$ en totonaque), les branches et les feuilles ce sont les différents villages et leurs comités locaux ». Ce discours est non seulement dirigé vers l'extérieur comme on a pu l'observer lors de réunions avec des organismes régionaux ou nationaux, mais aussi vers l'intérieur, c'est-à-dire en totonaque, pendant les séances de travail de l'OIT. Ainsi, le rapport d'identification aux arbres est mobilisé, cette fois, sur le plan politique ${ }^{26}$.

53 On peut donc synthétiser dans un tableau le système d'oppositions qui se dégage des descriptions de paysages par les Totonaques de Huehuetla :

\begin{tabular}{|l|l|}
\hline froid-frais & chaud \\
\hline
\end{tabular}


La présence ou l'absence d'arbres, en particulier, et de végétation feuillue (katuwan, litt. " là où il y a des feuilles »), en général, constitue donc un critère majeur dans la description des paysages ; elle traduit également des différences socio-économiques et culturelles. Elle est à son tour associée à l'opposition « chaud/froid (ou frais) » reproduisant dans la conception des espaces ce schème cosmologique. Ainsi, la compartimentalisation entre éléments indigènes et métis, absente sur le plan religieux entre rites indiens (ou considérés comme tels) et apports extérieurs, semble être reportée dans la typologie des espaces. Ce processus de compartimentalisation permet de construire la frontière entre les deux groupes selon les modalités analysées par Barth (1969).

Cependant, parmi les photos de paysages " totonaques ", se distinguent celles des ravins escarpés et des grottes pour lesquelles les commentaires sont plutôt négatifs : «Tu vois, c'est moche (lixkan) pour y marcher, il y a beaucoup de rochers ». En l'occurrence, le terme totonaque lixkan, généralement traduit en espagnol par feo ( « laid »), ne renvoie pas à un jugement esthétique, mais plutôt à une explication faisant référence au sens pratique, à la difficulté effective d'évoluer dans un fond de ravin accidenté. On peut sans doute extrapoler en disant que cette remarque fait également allusion aux différents dangers que l'on peut trouver dans ces lieux : perdre son âme à cause de l'effroi que les esprits peuvent provoquer, rencontrer des êtres antédiluviens pétrifiés (les gros rochers omniprésents en ces lieux) et susceptibles de devenir serpent ou «tigre » une fois la nuit tombée, attraper le « mauvais souffle » qui a une influence froide et provoque des troubles de l'âme et physiques (perte d'appétit, tremblements, etc.). D'après les Totonaques, ce type de maladie n'affecte pas les personnes vivant en ville, seulement celles qui habitent en des zones peu peuplées (Espadas et Zita 1982, p. 145). Ariel de Vidas (2002) a analysé une logique similaire concernant le discours nosologique des Teenek de la Huastèque veracruzaine : ceux-ci interprètent les différentes maladies en tenant compte de l’identité ethnique (certaines maladies étant considérées comme propres aux Teenek et non pas aux Métis).

56 En résumé, les éléments que nous avons abordés ici semblent situer le « nous » totonaque dans une position intermédiaire entre les " autres » métis, d'une part, et les « autres » sauvages - les esprits de la montagne -, d'autre part ; ou encore entre le monde « trop chaud » des paysages dénudés des pâturages, des bourgs et des villes sans arbres et celui " trop froid » des esprits de la forêt habitant ravins et grottes. Ces conclusions rejoignent l'analyse d'Ariel de Vidas (2002, p. 189) à propos du schéma dit « dualiste » du contraste " chaud/froid », en ce que celui-ci correspond plus à une relation dyadique qu'à une opposition dichotomique et permet un point de vue relativiste : les lieux considérés comme indigènes sont « froids » ou « frais » par rapport aux espaces métis, mais les lieux «sauvages » sont « froids » par rapport aux espaces « domestiqués » par les Totonaques. Ainsi, les espaces cultivés sous une couverture boisée ou arbustive (qu'elle soit permanente, comme pour la caféière, ou temporaire, comme pour la milpa) et considérés comme frais représentent un lieu idéal intermédiaire et représentatif de la bonne sociabilité totonaque, tout comme l'équilibre entre chaud et froid assure la bonne santé et l'esprit de mesure assure le « bien vivre».

Les arbres, ou du moins la végétation en tant que couvert forestier ou arbustif, établissent la médiation entre ces deux pôles. Le principe dyadique de la polarité chaud/froid semble donc être " répliqué » ${ }^{27}$ à tous les niveaux, de l'ordre cosmologique et temporel à la nosologie et à la classification des plantes. Ce schème de classification " dualiste » s'inscrirait-il dans un noyau central de concepts - ou dans ce que Carlsen et Prechtel (1991, p. 25) appellent « une construction normative formalisée »- qui guiderait les transformations de la configuration 
culturelle locale ? C'est ce que semble indiquer son application à l'interprétation identitaire des paysages dans les constructions discursives du rapport aux métis.

58 Remerciements : La recherche sur le terrain, qui a duré plusieurs mois sur une période de deux ans, a pu être réalisée grâce à une allocation de recherche qui m'a été attribuée pour faire une thèse au sein du CERMA/EHESS et grâce à une aide financière obtenue dans le cadre du programme " Aires culturelles » du ministère des Affaires étrangères. Je tiens à remercier Jacques Galinier, Philippe Descola, Emilia Velázquez, Aline Hémond, Odile Hoffman, Anath Ariel de Vidas et Esther Katz pour leurs commentaires et leurs conseils sur mes recherches, ainsi que Duna Troiani pour ses corrections, notamment celles concernant la transcription des passages en totonaque. Ma reconnaissance va aussi aux jeunes Totonaques du Centre d'Études Supérieures Indigènes Kgoyom (CESIK) de Huehuetla, à l'équipe d'ethnographes, dirigée par Hugo García Valencia de l'INAH-Xalapa, qui m'ont fourni de précieuses données sur les Totonaques du Veracruz, ainsi qu'à Miguel Ángel Martínez Álvaro de l’Institut de Biologie de l'UNAM pour les identifications botaniques et l'aimable transmission des travaux de son équipe.

\section{Bibliography}

DOI are automaticaly added to references by Bilbo, OpenEdition's Bibliographic Annotation Tool.

Users of institutions which have subscribed to one of OpenEdition freemium programs can download references for which Bilbo found a DOI in standard formats using the buttons available on the right.

Format

APA

MLA

Chicago

The Bibliographic Export Service is accessible via institutions subscribing to one OpenEdition freemium programs.

If you wish your institution to become a subscriber to one OpenEdition freemium programs and thus benefit from our services, please write to: access@openedition.org.

Format

APA

MLA

Chicago

The Bibliographic Export Service is accessible via institutions subscribing to one OpenEdition freemium programs.

If you wish your institution to become a subscriber to one OpenEdition freemium programs and thus benefit from our services, please write to: access@openedition.org.

ABERCROMbie Thomas

1990 « Ethnogenèse et domination coloniale », Journal de la Société des Américanistes, 76, pp. 95-104.

DOI : $10.3406 /$ jsa.1990.1359

Alcorn Janis B.

1984 Huastec Mayan ethnobotany, University of Texas Press, Austin.

\section{ApPadurai Arjun}

1995 "The production of locality », in R. Fardon (éd.), Counterworks: managing the diversity of knowledge, Routledge, Londres/New York, pp. 204-225.

\section{Aramoni Burguette María Elena}

1990 Talokan tata, talokan nana, nuestras raíces: hierofanías y testimonios de un mundo indígena, CONACULTA, Mexico. 


\section{ARIEL DE VidAs Anath}

2002 Le Tonnerre n'habite plus ici. Culture de la marginalité chez les Indiens teenek (Mexique), Éditions EHESS, Paris.

\section{BARTH Fredrik (éd.)}

1969 Ethnic groups and boundaries: the social organization of culture difference, Little, Brown and Company, Boston.

\section{Beaucage Pierre}

1997 « The traditional Indian coffee orchard of Eastern Mexico », Journal of Ethnobiology, 17 (1), pp. 45-68.

2000 « Fragmentation et recomposition des identités autochtones dans quatre communautés des régions caféicoles du Mexique », Recherches amérindiennes au Québec, 31 (1), pp. 9-19.

\section{Format}

APA

MLA

Chicago

The Bibliographic Export Service is accessible via institutions subscribing to one OpenEdition freemium programs.

If you wish your institution to become a subscriber to one OpenEdition freemium programs and thus benefit from our services, please write to: access@openedition.org.

\section{Blanco José L., Luisa Paré et Emilia Velásquez}

1992 «El tributo del campo a la ciudad: historias de chaneques y serpientes », Revista mexicana de sociología, 3, pp. 131-137.

DOI : $10.2307 / 3541011$

\section{BouTé Vanina}

2003 "Une tradition disputée : enjeux identitaires dans une communauté des Andes boliviennes ", Journal de la Société des Américanistes, 89-2, pp. 125-148.

Format

APA

MLA

Chicago

The Bibliographic Export Service is accessible via institutions subscribing to one OpenEdition freemium programs.

If you wish your institution to become a subscriber to one OpenEdition freemium programs and thus benefit from our services, please write to: access@openedition.org.

\section{Carlsen Robert. S. et Martin Prechtel}

1991 « The flowering of the dead: an interpretation of highland Maya culture », Man, n.s., 26, pp. 23-42.

DOI : $10.2307 / 2803473$

\section{Córdoba Olivares Francisco}

1968 Los totonacas de la región de Huehuetla, Pue., Tesis de maestría, Universidad Veracruzana, Xalapa.

\section{Format}

APA

MLA

Chicago

The Bibliographic Export Service is accessible via institutions subscribing to one OpenEdition freemium programs.

If you wish your institution to become a subscriber to one OpenEdition freemium programs and thus benefit from our services, please write to: access@openedition.org.

\section{Descola Philippe}

2001 « Par-delà la nature et la culture », Débat, 114, mars-avril, pp. 86-101.

DOI : 10.3917/deba.114.0086

\section{ELLISON Nicolas}

2004 Entre l'ombre des caféiers et la chaleur du maïs. Reproduction sociale, usages et représentations de l'environnement en pays 
totonaque, Sierra de Puebla (Mexique), Thèse de doctorat, École des Hautes Études en Sciences Sociales, Paris.

Espadas Resendiz M. et G. Zita Padilla

1982 Contribución al conocimiento de la flora medicinal de los totonacas de la Sierra de Puebla (Tuzamapan de Galeana, Puebla), Tesis de Biología, Universidad Nacional Autónoma de México, Mexico.

\section{FosT ER George M.}

1978 " Hippocrates' latin american legacy: "hot" and "cold" in contemporary folk medecine », in R. K. Wetherington (éd.), Colloquia in anthropology, Southern Methodist University, Dallas, pp. 3-19.

\section{GALINIER Jacques}

1997 La Moitié du monde. Le corps et le cosmos dans le rituel des Indiens otomi, PUF, Paris.

\section{Garavaglia Juan Carlos}

1995 « Atlixco : l'eau, les hommes et la terre dans une vallée mexicaine, Xve-Xvire siècle », Annales. Histoire et Sciences Sociales, 6 , pp. 1319-1349.

\section{GruZINSKI Serge}

2004 Les Quatre parties du monde. Histoire d'une mondialisation, Éditions de La Martinière, Paris.

\section{ICHON Alain}

1969 La Religion des Totonaques de la Sierra, Éditions du CNRS, Paris.

1973 La religión de los totonacas de la Sierra, Instituto Nacional Indigenista, Mexico.

Jiménez Ávila E. et A. Gómez Pompa (éds)

1982 Estudios ecológicos en el agroecosistema cafetalero, Continental, Mexico.

\section{KATz Esther}

1991 « Représentation de l'environnement et identité chez les Mixtèques du Mexique », Écologie humaine, IX (2), pp. 25-37.

2000 «Fleurs de café, luttes de pouvoir : évolution de la caféiculture en pays mixtèque (Mexique) », in Jean-Christian Tulet et Jacques Gilard (éds), La Fleur du café, Karthala, Paris, pp. 165-188.

\section{López Austin Alfredo}

1996 Cuerpo humano e ideología. Las concepciones de los antiguos nahuas, I, Universidad Nacional Autónoma de México, Mexico. 1997 Les Paradis de brume. Mythes et pensée religieuse des anciens Mexicains, IHEAL/ Maisonneuve et Larose, Paris.

\section{Lovell Nadia (éd.)}

1998 Locality and belonging, Routledge, Londres/New York.

\section{McQuown Norman (éd.)}

1990 Arte de la lengua totonaca, UNAM, Mexico [auteur anonyme].

\section{MünCH Galindo Guido}

1992 « Acercamiento al mito y sus creadores », Anales de Antropología, 29, pp. 285-299, Instituto de Investigaciones Antropológicas, UNAM, Mexico.

\section{NAZAREA Virginia}

1999 «Lenses and latitudes in landscapes and lifescapes », in Virginia Nazarea (éd.), Ethnoecology: situated knowledge/located lives, University of Arizona Press, Tucson, pp. 91-106.

\section{Rival Laura}

1998 "Trees, from symbols of life and regeneration to political artefacts ", in Laura Rival (éd.), The social life of trees. Anthropological perspectives on tree symbolism, Berg, Oxford/New York, pp. 1-38.

\section{ROGER Alain}

1997 Court traité du paysage, Gallimard, Paris. 


\section{STRESSER-PÉAn Guy}

1998 Los lienzos de Acaxochitlán (Hidalgo) y su importancia en la historia del poblamiento de la Sierra Norte de Puebla y zonas vecinas, Gobierno del Estado de Hidalgo/CEMCA, Mexico.

\section{Toledo Victor Manuel, Julia Carabias, Cristina Mapes et Carlos Toledo}

1993 Ecología y autosuficiencia alimentaria. Hacia una opción basada en la diversidad biológica, ecológica y cultural de México, Siglo Veintiuno, Mexico.

\section{Troiani Duna}

2003 Aperçu grammatical du totonaque de Huehuetla, Puebla, Mexique, LINCOM, coll. "Studies in Native American Linguistics » 49, Munich.

\section{ViEILLESCAZES Alain}

1983 Ethnobotanique des Indiens totonaques de la Sierra Norte de Puebla (Mexique), Thèse de doctorat, Université Pierre et Marie Curie, Paris.

\section{VogT Evon Z.}

1970 The Zinacantecos of Mexico : a modern Maya way of life, Holt, Rinehart and Winston, New York/Chicago/San Francisco.

Format

APA

MLA

Chicago

The Bibliographic Export Service is accessible via institutions subscribing to one OpenEdition freemium programs.

If you wish your institution to become a subscriber to one OpenEdition freemium programs and thus benefit from our services, please write to: access@openedition.org.

\section{WACHTEL Nathan}

1992 « Note sur le problème des identités collectives dans les Andes méridionales », L'Homme, 122, pp. 39-52.

DOI : 10.3406/hom.1992.369522

\section{Williams García Roberto}

1963 Los tepehuas, Universidad Veracruzana, Xalapa.

\section{Notes}

1 L'enquête de terrain a été effectuée dans six villages du municipe de Huehuetla, principalement à Lipuntahuaca, sur une période de dix-huit mois répartis entre février 2000 et mars 2002.

2 Le municipe de Huehuetla est devenu accessible, grâce à une piste carrossable, au cours de la seconde moitié des années 1980 . Les commerçants métis, qui monopolisaient le commerce muletier, s'opposèrent longtemps à la construction d'une véritable route reliant Huehuetla au réseau routier du haut plateau. Depuis 2001, cette piste est goudronnée et deux liaisons quotidiennes de bus relient le municipe à la capitale de l'État (Puebla). La durée du trajet n'est plus que de six heures.

3 Comme c'est aujourd'hui le cas dans la majorité des communautés totonaques et nahua de la Sierra de Puebla, ce système de charges est limité au domaine religieux. Les charges civiques traditionnelles ont disparu au profit des structures administratives locales officielles dont les postes sont le plus souvent occupés par des Métis. Cet état de fait est toutefois remis en cause dans plusieurs municipes - dont Huehuetla - où de nouvelles organisations indiennes luttent pour le pouvoir local.

4 Sans affirmer une origine purement mésoaméricaine du principe dualiste chaud/froid - que l'on retrouve d'ailleurs un peu partout dans le monde, mais rarement avec la même place centrale dans les représentations cosmologiques - je suis volontiers l'argument que López Austin (1996, pp. 300-317) a développé à l'encontre de l'hypothèse de Foster sur l'origine uniquement européenne du dualisme chaud/froid : une si rapide et profonde adoption de cet élément de la théorie des humeurs ne peut être 
expliquée que parce qu'il faisait écho à un certain nombre de conceptions indigènes. Il n’y a pas par ailleurs, à mon avis, de contradiction entre cet argument et celui de Gruzinski (2004) à propos des métissages complexes entre les conceptions médicales de l'Ancien Monde - le système d'Hippocrate et de Galien - et les connaissances thérapeutiques indigènes au cours du premier siècle de domination coloniale, argument qui mène cet auteur à affirmer l'existence d'une « mondialisation » des savoirs dès cette époque. Il est donc fort probable que la « théorie des humeurs », importée par les Européens, recoupait une conception dualiste indigène du chaud et du froid appliquée au domaine cosmologique, lui-même étroitement lié aux conceptions préhispaniques sur la santé humaine.

5 Cette opposition est aussi fort commune dans les Andes où elle a été relevée et analysée par Abercrombie (1990), Wachtel (1992) et Bouté (2003) entre autres.

6 À la suite des changements politiques dans le rapport de force entre Indiens et Métis, au cours des années 1990, mais aussi en raison de l'arrivée d'un nombre croissant de personnes issues des villes (travaillant pour l'Institut national indigéniste (INI), dans les cliniques, les écoles, les ONG, etc.), les expressions euphémiques « gente de rancho », « gente de comunidad » ou «indígenas » sont de plus en plus usitées par les Métis du bourg pour désigner les habitants totonaques des villages et hameaux périphériques.

7 Cette taille est modeste comparée à d'autres régions du Mexique mais, à l'échelle locale de Huehuetla, les ganaderos correspondent à des terratenientes, c'est-à-dire à de " grands propriétaires ». Sur l'ensemble du municipe, seulement une dizaine de ménages totonaques possède entre 2 et 10 vaches. En revanche, plusieurs ganaderos de la bourgade ont des pâturages, de superficie parfois très grande (plusieurs centaines d'hectares), dans les municipes voisins et surtout dans les basses terres du Veracruz.

8 Comme je l'ai déjà signalé plus haut, la majorité des chefs de famille louent un terrain pour une récolte de maïs qui ne couvre, dans la plupart des cas, que la moitié de la consommation annuelle, alors qu'ils y consacrent plus de la moitié de leur temps de travail (Ellison 2004, pp. 420-449). Le travail relatif à la culture du maïs est socialement très valorisé, car il est l'occasion d'une entraide ritualisée qui actualise régulièrement les relations de solidarité, de parenté ou de voisinage. De plus, il s'insère dans un calendrier rituel et festif complexe, étroitement lié à la reproduction sociale de la communauté (ibid., pp. 349-351).

9 Voir aussi Alcorn (1984) pour les Teenek de la Sierra de Hidalgo ; Katz (2000) pour la région mixtèque ; Beaucage (1997, 2000) pour les Nahua de Cuetzalan.

10 En effet, l'anthropomorphisme du maïs, tel que le conçoivent les Totonaques, permet d'établir une équivalence entre le cycle de cette plante et la vie humaine, ce qui se répercute sur l'ensemble de la société : les phases du cycle du maïs coïncident avec les temps forts du calendrier religieux qui rythment la vie sociale. Les semailles et les récoltes sont l'occasion de réactualiser les liens sociaux, que ce soit à travers l'entraide autour du travail agricole ou pendant l'organisation des fêtes patronales.

11 L'association entre l'âme d'un mort récent et un type de mouche appelée kuxta est peut-être une réminiscence de la notion de double animal ou nahual. Selon un lexique établi par les franciscains de Hueytlalpan au xvie siècle, le mot totonaque kuxta désigne, d'ailleurs aujourd'hui encore, autant cet insecte que l'âme (McQuown 1990). L’idée que certains chamanes (skgoyona', plur. lakgskgoyonan, en totonaque ou brujos en espagnol) se transforment en bêtes féroces ou en boules de feu est présente dans les commentaires concernant les pratiques de " sorcellerie », mais comme un souvenir des temps révolus, c'est-à-dire 50 ans en arrière. Ce qui correspond au début de la néo-évangélisation des années 1950. De même, plutôt que l'explication par le déterminisme écologique de la déforestation avancée par Ichon (1973, p. 207), c'est sans doute cette domination de la religion catholique qui explique à Huehuetla l'abandon du tonalisme chez les Totonaques.

12 D’après le mythe de la naissance du soleil chez les Totonaques septentrionaux, pour aider le soleil à entamer son périple céleste, les oiseaux le criblèrent de flèches, d'où les gouttes de sang à l'origine des cultigènes (Ichon 1973, p. 67). Pour les Tepehua de Pisaflores, c'est à partir du sang provenant de l'annulaire du soleil, coupé par les étoiles, que naissent toutes les plantes (Williams García 1963).

13 J'ai enregistré une trentaine de récits en langue vernaculaire. Ils ont pu être traduits en espagnol grâce à l'aide de jeunes Totonaques bilingues. Pour chaque récit, j’ai comparé deux traductions en espagnol avec le texte original, ce qui m'a permis d'en faire la traduction en français.

14 Dans le nord de la Sierra, San Juan Aktsini’ est décrit comme étant chasseur, paresseux et grand buveur - traits qu'il a en 
commun avec Tlaloc, la divinité aztèque de la pluie, qui était aussi le dieu de l'ivresse (Ichon 1969).

15 Au sujet du Tlalocan chez les anciens Nahua, voir notamment López Austin (1997). En ce qui concerne les représentations du "Talokan » parmi les Nahua contemporains, voisins des Totonaques dans la région de Cuetzalan, voir Aramoni (1990, pp. 79-146).

16 Elisabeth Perralta (INAH-Xalapa), communication personnelle.

17 Ce détail marque bien le lien de cet esprit avec le monde «non culturel », sauvage : les champignons (qui, dans l'alimentation totonaque, agrémentent parfois les bouillons) ne sont pas considérés comme un aliment véritable, ils poussent sur les arbres (donc dans la forêt) et ne sont pas cuisinés. Chez les Totonaques septentrionaux, le maître de la forêt, Aksanjan, est l'esprit tutélaire gardien des champignons et des ruches sylvestres (Ichon 1973, p. 154).

18 Dans les contes concernant le maitre des animaux, pour réparer ses prélèvements excessifs, le chasseur séquestré devra s'occuper des animaux, à moins de n'être livré en pâture aux pécaris. Dans les villages de Huehuetla, on m’a rapporté des cas de personnes, connues pour leur goût pour la chasse, dont on explique la maladie ou le décès à cause d'une rencontre avec le Xmalana Takgalhinin.

19 Un autre fait permet d'établir la parenté entre Jean de la Forêt et San Juan Aktsini'. Ce dernier est souvent appelé Juan Loso, sans doute par contraction avec Juan del Oso, faisant référence à la tradition européenne de Jean de l'Ours, comme esprit de la forêt (je remercie Duna Troiani de m'avoir signalé ce rapprochement). Ce conte, dont une version existe dans la plupart des traditions orales européennes, évoque les épopées d’un géant mi-homme mi-ours, né d’une fille enlevée par un ours. Il existe à Huehuetla une tradition orale qui en est sans doute inspirée - ou qui fusionne ce conte avec une tradition indigène parallèle - et où l'ours est remplacé par un singe (Troiani 2003). Cette narration acquiert toutefois une profondeur symbolique particulière dans le contexte cosmologique mésoaméricain car, dans la tradition totonaque, les hommes antédiluviens avaient été transformés en singes et les grottes représentaient des lieux de communication avec ces ancêtres.

20 Le terme totonaque skga'wiwi signifie indifféremment « froid », à connotation néfaste, et « frais », à connotation bénéfique.

21 Vieillecazes (1983, p. 49) signale d'ailleurs aussi que les Totonaques de Tuzamapan (distant de seulement une vingtaine de kilomètres de Huehuetla) classifient les plantes selon les mêmes critères - exposition au soleil et teneur en eau - et considèrent la forêt, le sous-bois et les animaux sauvages comme « froids ».

22 Ce qui est une constante mésoaméricaine, ainsi qu'en témoignent les représentations du Tlalocan, perçu comme inframonde sylvestre parmi les anciens Nahua (López Austin 1997, pp. 211-214). La relation entre le Talokan, source des richesses terrestres pour les Nahua contemporains de Cuetzalan, et le maître de la forêt Juan del Monte ou Tepeyolo, « cœur ou esprit de la montagne », manifeste cette continuité. De même, les Tzotzil des hautes terres du Chiapas font directement correspondre les bois sacrés avec les montagnes des ancêtres (Vogt 1970, pp. 5-6, 10-11) ; chez les Mixtèques, il existe aussi une continuité entre la forêt et l'inframonde : le terme yuku désigne à la fois la forêt et la montagne dans laquelle se trouvent les grottes (Katz 1991, p. 29).

23 Je me suis inspiré pour cela de Nazarea (1999) et de la méthode des tests d'aperception, sans prétention de représentativité statistique.

24 En l'absence d'une tradition picturale locale (à la différence d'autres régions indiennes du Mexique) et étant donné la généralisation tardive de la scolarisation, on peut exclure toute « artialisation » du pays en paysage, dans le sens proposé par Roger (1997, pp. 17-20). Toutefois, les manuels scolaires bilingues, introduits au cours des années 1990, diffusent désormais des photographies de la Sierra et, par-là, certains canons esthétiques (au sens occidental de l'objectification de la nature).

25 Ce terme, littéralement "eau-montagne », correspond au mot aztèque altepetl qui se traduit de la même façon. Cette expression évoque l'identification de la communauté avec la montagne des ancêtres.

26 Sur le symbolisme des arbres utilisés pour rendre plus concrète la notion de vie sociale dans différentes cultures et sur l'arbre comme « objet politique », voir Rival (1998). Concernant l'arbre comme pilier du monde dans la cosmologie mésoaméricaine, voir López Austin (1997, pp. 19-22). L'« Arbre fleuri » du paradis mythique Tamoanchan des anciens Nahua est d'ailleurs associé au symbolisme des contraires, notamment à l'opposition chaud/froid (ibid., pp. 110-114). 
27 Selon l'expression de Vogt (1970, pp. 100-108) qui l'applique à la reproduction de structures identiques aux différents niveaux de l'organisation sociale des Tzotzil de Zinacantan, mais aussi à la réitération de certains concepts dans différents contextes de la vie sociale.

\section{List of illustrations}

Caption Fig. 1 - Graphique sur l'évolution de l'usage des sols en hectares, municipe de Huehuetla, $1960-2000$.

URL http://jsa.revues.org/docannexe/image/1332/img-1.jpg

File image/jpeg, 28k

Caption Fig. 3 - Les images de San Salvador et de San Miguel en procession lors de la fête patronale de

Huehuetla (cliché Ellison).

URL http://jsa.revues.org/docannexe/image/1332/img-2.png

File image/png, 779k

Caption Fig. 4 - Maison d'un éleveur de bétail, bourgade de Huehuetla (cliché Ellison).

URL http://jsa.revues.org/docannexe/image/1332/img-3.jpg

File image/jpeg, 180k

Caption Fig. 5 - Pâturage (en bas), milpas fraîchement sarclées et caféière, Huehuetla-Leakaman (cliché Ellison).

URL http://jsa.revues.org/docannexe/image/1332/img-4.jpg

File image/jpeg, 283k

\section{References}

Bibliographical reference

Journal de la Société des Américanistes, 2004, 90-2, pp. 35-62

Electronic reference

Nicolas Ellison, « Une écologie symbolique totonaque. Le municipe de Huehuetla (Puebla, Mexique) », Journal de la société des américanistes [Online], 90-2 | 2004, Online since 05 June 2009, connection on 09 March 2016. URL : http://jsa.revues.org/1332; DOI : 10.4000/jsa.1332

\section{About the author}

\section{Nicolas Ellison}

Centre de recherche sur les mondes américains, CERMA, UMR 8565, Paris/Équipe de recherche en ethnologie amérindienne, EREA, CNRS, Villejuif [nellison@ehess.fr] 


\section{Copyright}

(C) Société des Américanistes 